\title{
Décadrages Décadrages
}

cinéma, à travers champs Cinéma, à travers champs

19 | 2011

Autour d'Elephant de Gus Van Sant

\section{L'œil d'Elephant : l'espace d'un regard}

Alain Boillat

Q OpenEdition

Journals

Édition électronique

URL : https://journals.openedition.org/decadrages/308

DOI : 10.4000/decadrages.308

ISSN : 2297-5977

\section{Éditeur}

Association Décadrages

\section{Édition imprimée}

Date de publication : 10 octobre 2011

Pagination : 48-69

ISBN : 978-2-9700668-3-5

ISSN : 2235-7823

Référence électronique

Alain Boillat, «L'œil d'Elephant : l'espace d'un regard », Décadrages [En ligne], 19 | 2011, mis en ligne le 10 octobre 2012, consulté le 03 avril 2022. URL : http://journals.openedition.org/decadrages/308 ; DOI : https://doi.org/10.4000/decadrages.308

(B) Décadrages 
1 Les informations parafilmiques jouent à cet égard un rôle clé puisque, dans le film, aucun anthroponyme ni toponyme n'assure l'établissement de ce référent précis - Elephant porte sur les adolescents américains à l'aube du XXI ${ }^{e}$ siècle, non sur la fusillade de Columbine en particulier. Le fait que l'un des tueurs se prénomme Eric pourrait laisser penser qu'il s'agit là d'un renvoi à l'une des personnes réelles ayant participé au drame, Eric Harris; cependant, les prénoms des personnages - dont les noms de famille demeurent significativement inconnus, les adolescents étant montrés dans leur solitude, disjoints de leur cercle familial - sont en fait simplement, pour la plupart, ceux des acteurs du film (majoritairement non professionnels): ainsi l'acolyte du personnage interprété par Eric Deulen ne s'appelle pas Dylan, mais Alex, comme l'acteur Alex Frost. Ce principe onomastique est révélateur de la démarche de Van Sant, dont le film "documente" bien plus la mise en scène qu'il occasionne que le fait divers. Ce que Van Sant dit des acteurs de son film précédent, Gerry (2002), vaut tout autant pour Elephant: "[...] j'ai eu envie qu'ils utilisent ce qu'ils sont dans la vie, qu'ils réagissent aux situations par euxmêmes, et surtout qu'ils ne construisent pas de personnages de fiction. [...] lls ont une psychologie, mais elle n'est pas exposée de façon didactique. Le personnage n'existe pas antérieurement à la personne qui l'incarne à l'écran" "Gus Van Sant - Roi du désert", Les Inrockuptibles, 3 mars 2004, cité par Edouard Arnoldy, Le cinéma entre les nuages, Crisnée, Yellow Now, 2009, p. 43).

2 En dépit de la juxtaposition de "voix narratives" (nous passons d'un personnage à l'autre), précisons qu'aucun personnage n'accède à une manifestation en voix over qui serait l'indice d'une certaine maîtrise énonciative. Au contraire, les manifestations vocales (in ou off) des protagonistes sont plutôt rares (l'un d'eux, prénommé Benny, ne prononce pas un seul mot), et les paroles tendent à se perdre dans le brouhaha ambiant. A cet égard, l'ancrage diégétique d'une musique au piano tout d'abord appréhendée comme extradiégétique - voir à ce propos l'article de Gaspard Vignon - joue un rôle important: cette emprise d'un personnage sur le discours filmique s'effectue significativement sur le plan des affects associés à la mélodie, non par le biais de l'expression verbale.

\section{L'œil d'Elephant: l'espace d'un regard}

\section{par Alain Boillat}

Au plan narratif, Elephant (USA, 2003) de Gus Van Sant présente une structure éclatée, témoignant d'une attirance pour la périphérie d'un drame qui, durant les deux premiers tiers du film, se dessine avant tout en filigrane, à travers les virtualités dont se compose l'horizon d'attente du spectateur lorsqu'il y reconnaît les événements tragiques du lycée de Columbine $\mathbf{1}$. Les menues actions du quotidien sont mises en exergue dans la continuité de leur déroulement, au détriment de l'organisation causale d'actions nodales. S'il s'agit d'un récit «polyphonique» dédié à la "voix" (narrative) 2 d'un certain nombre de protagonistes identifiés dans des intertitres par la mention de leur prénom, les personnages nous sont toutefois montrés avec distance et peuvent être considérés comme faiblement construits en regard des canons usuels de la "psychologie du personnage» prônés par les "gardiens» du temple hollywoodien. Confrontant le spectateur à la pure extériorité de jeunes individus figurés comme des silhouettes errantes dont les pensées et motivations nous demeurent inaccessibles, Elephant semble faire usage de certains procédés communément associés à une volonté d'intériorisation - en particulier le flash-back et une technique de filmage que l'on pourrait considérer a priori comme parente de la «caméra subjective» - avec un régime flottant d'indifférence, de non-immersion dans l'action. Cette forme de distance n'est pas celle qu'instaure le deus ex machina du cinéma classique hollywoodien en régulant la transmission des informations narratives : paradoxalement, Van Sant se situe au plus près de ses personnages, si près que leur opacité foncière nous paraît manifeste. Le cinéaste met en évidence cette particularité de son cinéma lorsqu'il examine dans un entretien les raisons de l'échec de son remake du film Psychose (Psycho, Alfred Hitchcock, USA, 1960), qu'il compare à l'original :

«Le principal fossé tient à la relation aux personnages: lui [Hitchcock] en fait des objets soumis à une énorme tension, toujours croissante. Le public, alors, entre dans un rapport d'intensité à distance avec les personnages. Dans mes films, au contraire, c'est comme si je devenais moi-même le personnage [...]. Les personnages que je 
filme, j'essaie de les regarder jusqu'au moment où je me confonds avec eux.»3

On pourrait croire en lisant ces propos que Van Sant prône une identification complète au personnage, mais il précise juste après qu'il est "un spectateur, un mateur», celui "qui regarde bouger des corps dans l'espace». C'est dans le creux qui s'ouvre entre un rapport fusionnel au personnage et la conscience du statut voyeuriste de la caméra qu'il s'agit à notre sens d'aborder Elephant.

En premier lieu, les procédés qui fonctionnent supposément au bénéfice de l'intériorisation méritent d'être examinés de plus près. En effet, il faut remarquer que les retours en arrière n'endossent dans Elephant guère de fonction explicative, dans la mesure où leur amplitude est le plus souvent très faible (quelques heures, voire quelques minutes) et où leur insertion vise principalement à assurer un changement de piste narrative, à permettre qu'un personnage donné puisse être relayé par un autre en tant que «cible» du regard de la caméra (avant, pour la plupart d'entre eux, de devenir la cible des tueurs). Notons toutefois qu'une certaine hiérarchie est établie entre les protagonistes qui résulte en partie des modalités du flash-back. En effet, si chaque introduction d'un nouveau personnage (à l'exception de Benny) momentanément présenté comme "central» est accompagnée d'une analepse interne, l'unique retour en arrière remontant à une période antérieure à la journée du massacre est consacré aux (futurs) assassins, Eric et Alex ${ }^{4}$. Par conséquent, contrairement à ce qui fut fréquemment mis en évidence à la sortie d'Elephant afin de valoriser le film pour sa "finesse» par rapport au documentaire à thèse de Michael Moore (Bowling for Columbine, USA, 2002) sorti l'année précédente, le film de Van Sant n'est pas dépourvu de toute dimension explicative dans sa façon de nous conduire à la fusillade: les humiliations subies à l'école, l'absence totale de culture historique (patente dans les commentaires d'Alex et de son complice à propos d'un documentaire sur le nazisme diffusé à la télévision), l'influence néfaste des jeux vidéo, l'achat aisé d'armes via internet $\mathbf{5}$ - couplé à une démission des adultes par rapport à leurs responsabilités de parents 6 -, voire le refoulement de l'homosexualité constituent autant de causes possibles du méfait ${ }^{7}$. Certes, ces différents aspects sont évoqués en passant, sans mise en exergue, ce qui contraste fortement avec la thèse unique à laquelle Moore subordonne l'intégralité de son film - celle qui consiste à voir dans le climat de peur et l'idéologie de l'autodéfense sciemment entretenus par les lobbys de l'industrie de l'armement la source des maux en question. Il faut néanmoins convenir que les tueurs bénéficient dans Elephant d'un traitement particulier visant à exposer leurs conditions

3 Olivier Joyard et Jean-Marc Lalanne, "Gus Van Sant. Je suis comme Colombo, je fais semblant de ne pas savoir", Cahiers du cinéma, n 579 , mai 2003, p. 24.

4 Ces deux personnages occasionnent également l'unique série de plans en flash-forward du film. II s'agit d'images qui, lors de la planification de la prise d'assaut du lycée, visualisent les différentes phases évoquées par les deux adolescents, selon une pratique très courante dans les films de gangsters ou de guerre (préparation d'un casse ou d'une offensive). La saccade et le choc qu'ils provoquent contrastent avec les longs plans-séquences qui, ailleurs dans le film, insufflent un rythme régulier: cet effet disruptif est un signe avant-coureur de l'irruption de l'horreur dans le quotidien.

5 Comme Michael Moore, Van Sant fait référence au fétichisme américain pour les armes puisque, dans la discussion entre John et son père qui ouvre le film, le seul point d'entente consiste à aller chasser ensemble en utilisant un modèle précis de fusil dont le père fait I'historique.

6 Notons que les parents sont presque systématiquement rejetés hors-champ dans les rares scènes où ils apparaissent.

7 La séquence de la réunion de la gay straight alliance fait écho à la relation entre Eric et Alex. 
8 Hormis le personnage de John (voir note 29).

9 Voir à ce propos Stéphane Bouquet et JeanMarc Lalanne, Gus Van Sant, Paris, Cahiers du cinéma, 2009, p. 148, note 2. Les auteurs font notamment remarquer que la protagoniste de ce film d'Ackerman où la violence surgit également de façon soudaine au sein du quotidien écoute à la radio la Lettre à Elise de Beethoven que l'Alex d'Elephant joue au piano.

$10 \mathrm{~A}$ propos de Zavattini, voir Francesco Casetti, Les théories du cinéma depuis 1945, Paris, Nathan, 1999 [1993], pp. 29-32.

11 Voir à ce propos l'article de Selim Krichane dans le présent dossier.

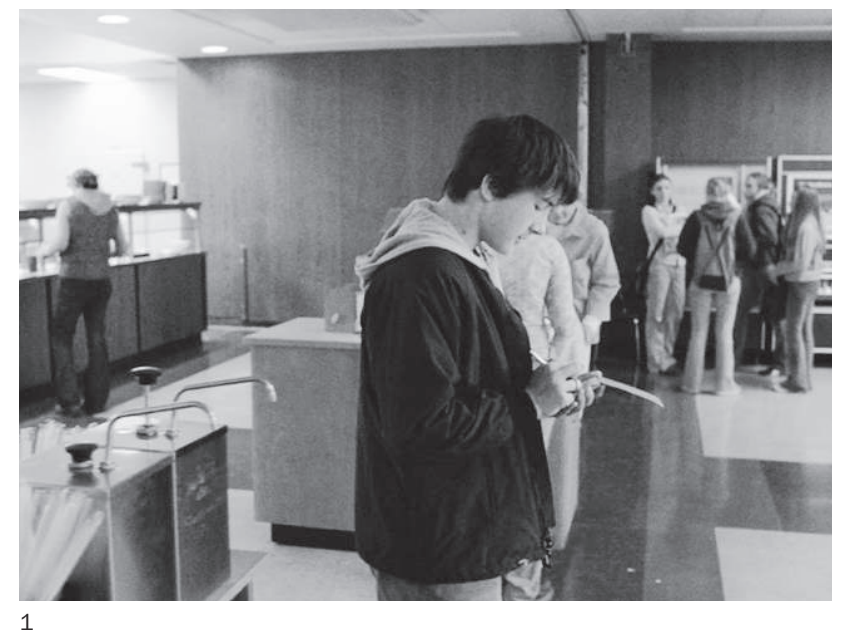

de vie, alors que les autres personnages ne sont jamais montrés ailleurs qu'au lycée 8 .

Quant au filmage, caractérisé par de longs déplacement de l'appareil effectués à l'aide d'une steadicam (dont l'opérateur est parfois placé sur un chariot très mobiles), nous pourrions dire qu'il se rapproche de l'esthétique de la filature telle que la prônait le critique et scénariste italien Cesare Zavattini, l'un des principaux théoriciens du «néoréalisme» pour qui le cinéma devait prendre pour objet les moments quelconques du quotidien (en excluant toute morale) - on sait que Van Sant s'est réclamé pour Elephant de l'influence du film Jeanne Dielman de Chantal Akerman (Belgique/France, 1975) ${ }^{\mathbf{9}}$-, livrer un récit basé sur la seule filature (pedinamento), dans la durée, d'un personnage au gré de ses vagabondages et rencontres ${ }^{\mathbf{1 0}}$. Cette dimension dédramatisante, quasi documentarisante se conjugue singulièrement dans Elephant avec un mode de captation qui emprunte certains procédés aux représentations «hyperfictionnelles» des nouveaux médias dont l'essor est contemporain de la sortie du film 11. Gus Van Sant opère ainsi une fusion entre le cinéma dit «d'art et essai» et la culture de masse qui, nous le verrons, n'est pas sans conséquences sur le traitement du «point de vue». Bien qu'il entende décrier les comportements déviants résultant de la consommation de nouveaux médias - les jeux vidéo sont présentés dans Elephant (ne seraitce qu'en raison de la place qui leur est octroyée dans le développement du récit) comme un facteur d'aliénation de l'individu -, Gus Van Sant ne s'interdit paradoxalement pas d'en ingérer certaines composantes, comme il le fait pour l'ensemble de la culture médiatique dans laquelle il baigne. 


\section{Caméra arpenteuse et mosaïque cartographique}

Les parcours présentés dans Elephant pour chacun des personnages individualisés ne sont pas prioritairement d'ordre narratif (on n'y trouve pas de clôture du récit, de processus de transformation de la caractérisation initiale, etc.) ni même sociologique (comme le serait l'étude de parcours de vie). Ils sont en fait appréhendés dans un sens tout à fait littéral: celui de la monstration, par l'image animée, d'un trajet effectué par un personnage - ce qui n'exclut pas que les attitudes de l'acteur/personnage en mouvement ou les lieux de départ et d'arrivée soient signifiants sur un plan sociologique. La dispersion, voire l'évanescence de potentiels foyers de focalisation est en quelque sorte compensée par un recentrement sur un espace-temps donné, arpenté en tous sens par les personnages et par l'appareil de prise de vues qui les prend en filature. En effet, les facettes de l'histoire racontée sont totalement chevillées à la topographie du lieu du fait divers, envisagé précisément dans la diversité et la banalité des actions qui le composent. L'importance de la configuration spatiale des lieux est d'ailleurs soulignée à plusieurs reprises dans le film : Alex effectue, un calepin à la main, des «repérages" dans le réfectoire (fig. 1), l'un des principaux lieux du massacre à venir; il utilise ensuite, pour planifier le méfait et se concerter avec son complice, une représentation schématique de l'établissement scolaire, sorte de plan d'aménagement. A l'instar des tueurs, Van Sant cartographie l'espace du lycée pour mieux y déambuler au gré de longs déplacements réalisés à l'aide d'une steadicam. Ces mouvements d'appareil, aériens, sont à la fois fortement vectorisés par les allées ou couloirs, et flottants dans le rapport de proximité qu'ils entretiennent avec les personnages. En juxtaposant
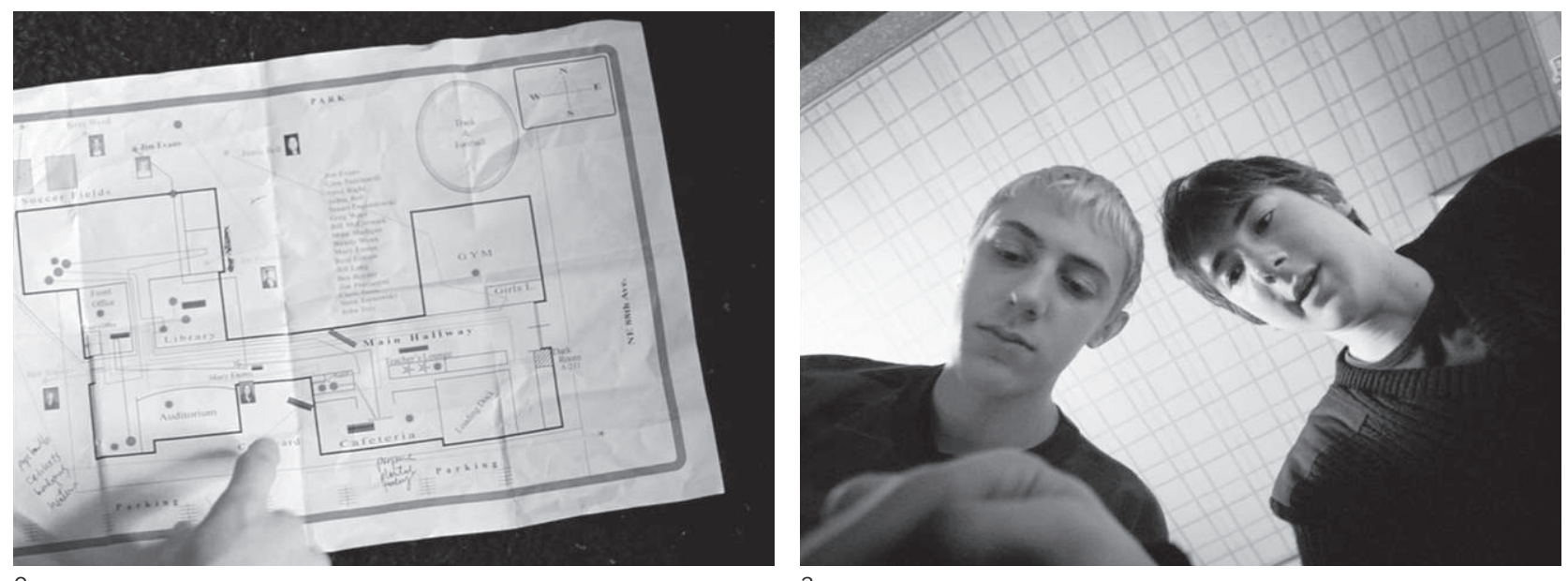
12 Documents diffusés en 2000 par CNN et disponibles sur http://edition.cnn.com/SPECIALS/ 2000/columbine.cd/Pages/DIAGRAM_TOC.htm. Pour la couverture du présent numéro, nous nous sommes inspirés des plans d'aménagement disponibles sur ce site.

13 Avec la généralisation de CCTV (postes de télévision en circuit fermé) et d'autres outils technologiques, il est évident que le but des autorités est d'assurer cette maîtrise de l'espace en amont afin d'anticiper toute action répréhensible. Mike Davis a relevé dans l'un de ses essais socio-historiques sur les métropoles états-uniennes que "l'ordre disciplinaire " examiné par Foucault s'applique désormais également aux établissements scolaires américains. II note par exemple que, "dans une école élémentaire de Santa Monica, on entraîne régulièrement les enfants à des 'exercices de fusillade." (Mike Davis, Au-delà de Blade Runner. Los Angeles et l'imagination du désastre, Paris, Allia, 2010 [1996], p. 54). II est évident que des drames tels que celui du lycée de Columbine (postérieur à la parution de l'ouvrage de Davis) peuvent servir à légitimer un tel contrôle policier. Le film Elephant se prêterait tout à fait à une utilisation didactique visant à entraîner de jeunes spectateurs en présentant différentes attitudes de victimes potentielles. dans une même représentation les trajectoires de plusieurs protagonistes, le cinéaste quadrille le lieu du drame, ainsi que le suggère la mise en évidence, sur le plan plastique, de surfaces en damiers (comme le motif du plafond situé à l'arrière-plan du contrechamp sur Eric et Alex lorsqu'ils sont penchés sur le plan d'aménagement du lieu, répétant une dernière fois les phases de leur projet sanglant, fig. 2-3).

Il faut de plus noter que la police du comté de Jefferson a réalisé, pour reconstituer le déroulement de la fusillade du 20 avril 1999 au Lycée de Columbine, près d'une centaine de "schémas d'activité» (activity diagramms) qui rendent compte, à différents moments, de l'emplacement exact des deux tueurs Klebold et Harris, des victimes et des témoins, ainsi que des actions des différents acteurs du drame ${ }^{\mathbf{1 2}}$. Cette segmentation associée à des espaces spécifiques (les "crime scenes») et son principe d'organisation de type topographique - chaque schéma est consacré à un sous-espace inscrit dans l'un des grands ensembles: l'extérieur et le parking, le niveau inférieur de l'immeuble, la cafétéria, la bibliothèque et le département des sciences - nous semblent également à l'œuvre dans la composition en mosaïque d'Elephant, qui constitue à cet égard une sorte de "simulateur", d'application en trois dimensions de ces instruments qui servent, par l'abstraction, à rendre le réel rétrospectivement lisible et appréhendable de façon panoptique ${ }^{13}$. Il est à ce titre significatif que l'édition MK2 du film sur support DVD ait opté pour un menu interactif organisé en fonction des parcours effectués par les différents personnages, le tracé de leurs déplacements apparaissant en couleur sur la reproduction, à l'écran, d'un croquis de la high school et de ses environs esquissé par Gus Van Sant, ainsi qu'en témoigne la signature du

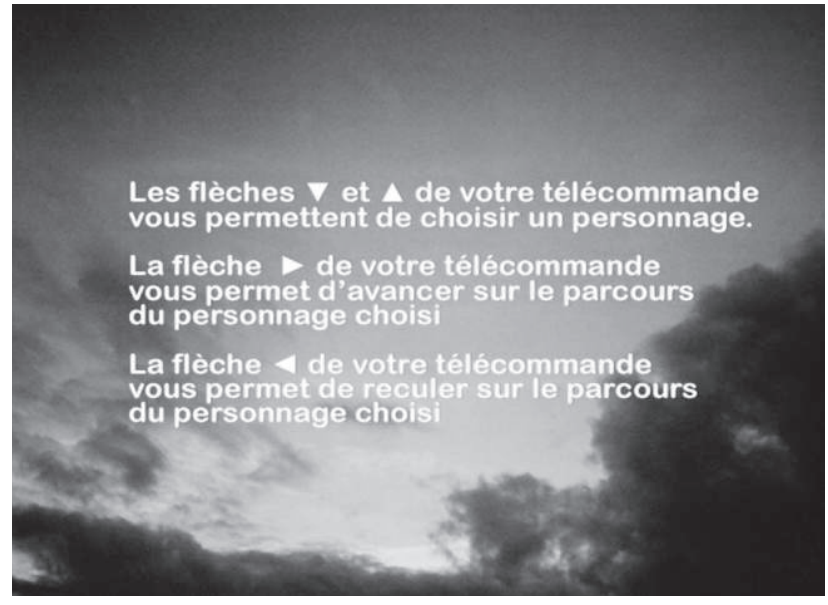

4

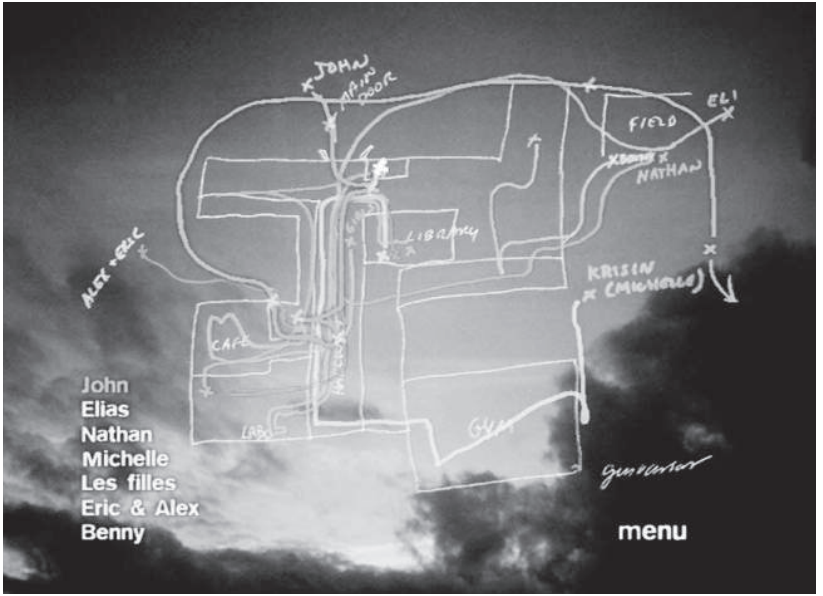

5 
cinéaste apposée dans le bord droit de l'image (fig. 4-5). Cependant, la représentation spatiale d'Elephant ne tient pas de la vue aérienne en plongée sur l'établissement: Van Sant opte pour une approche immersive en filmant à hauteur d'homme, tandis que le ciel se présente comme l'horizon du regard.

Dans le rapport de police, les cartes constituent le pendant spatial d'une chronologie des faits établie avec une extrême minutie. Elle est présentée ainsi dans le document disponible sur le site de CNN:

"La chronologie a été mise à jour à de nombreuses reprises en fonction de l'obtention de nouvelles informations auprès des différentes sources. Le résultat se présente sous la forme d'une chronologie PRIMAIRE [PRIMARY timeline] offrant un compte rendu sommaire des événements du 20 avril 1999: les mouvements de Eric Harris, Dylan Klebold et des forces de police, l'organisation de la situation d'urgence et la couverture médiatique. Cette chronologie constitue la base d'une chronologie NARRATIVE [NARRATIVE timeline] plus détaillée et complète des événements qui est comprise dans le présent rapport. »14

La mise en scènes (au sens d'une série de crime scenes) proposée par Gus Van Sant s'inscrit dans une démarche similaire à l'établissement de la primary timeline évoquée dans ce rapport, qui se situe en deçà d'une opération de narrativisation. La compilation de sources provenant d'un grand nombre de témoins donne lieu dans Elephant à une structure polyphonique, suite d'éclairages fragmentaires qui dépendent de l'emplacement des protagonistes à un moment donné. Comparativement aux différents «acteurs" du drame mentionnés dans l'introduction de ce rapport, ce que Gus Van Sant nous donne à voir est très restreint: tout ce qui concerne les interventions extérieures - polices, médias, secours, etc. - est occulté au profit d'une vision de l'intérieur qui émane exclusivement des adolescents. En fait, on pourrait dire que le regard de la société sur l'événement est déplacé dans l'acte même de filmer (d'où, sans doute, ce mélange d'intériorisation et de mise à distance) : la «couverture médiatique» est ici prise en charge par le cinéma de fiction selon des procédures qui lui sont propres, notamment la création de personnages. La démarche du cinéaste - qu'il s'agit ici de concevoir de façon physique, comme un rythme de déplacement - est similaire à celle des tueurs qui entendent maîtriser l'espace et viser, apparemment au hasard et de façon désinvolte, ceux qui entrent dans leur champ de vision. A ce titre, Elephant actualise en quelque sorte dans sa genèse et son mode de filmage la coprésence que l'on trouve sur l'affiche de Bowling for Columbine (Michael Moore, 2002) d'une caméra et d'une arme à feu sur
14 http://edition.cnn.com/SPECIALS/2000/ columbine.cd/Pages/TOC.htm. Ce document porte le sceau "Sheriff. Jefferson County, colorado". 


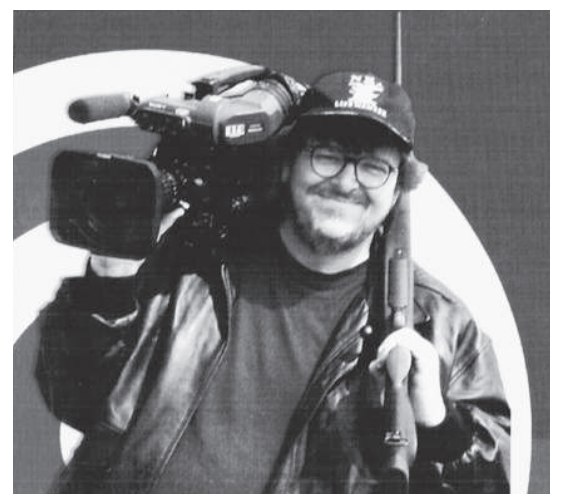

6

15 Paul Virilio, Guerre et cinéma. Logistique de la perception, Paris, Editions de l'Etoile/ Cahiers du cinéma, 1984.

16 Mes remerciements à Adeena Mey pour la suggestion de cette filiation, qui mériterait un examen plus approfondi.

17 Pour un exemple de ce type d'identification assortie d'une exploration d'un espace, voir Dominic Arsenault et Bernard Perron, "L'empire vidéoludique: comment les jeux vidéo ont conquis l'univers de Star Wars", Décadrages, no 8-9, automne 2006, pp. 101-102.

18 Voir dans le présent dossier l'article de Selim Krichane (fig. 6, et le commentaire qui lui est associé).

19 On trouvait déjà ce mode de figuration dans Clockers de Spike Lee (1995), où le jeune assassin se déplace sur sa bicyclette en direction de sa cible tel son avatar dans un jeu de réalité virtuelle. les épaules d'un cinéaste qui entend tirer à boulets rouges sur les défenseurs des lobbys de l'armement (fig. 6). D'ailleurs, cette association entre la prise d'images et l'industrie de l'armement, qui fut discutée dans ses fondements idéologiques par Paul Virilio à propos du cinéma ${ }^{\mathbf{1 5}}$, peut être envisagée de façon plus large, notamment en termes d'archéologie des médias: du "fusil photographique» conçu par Etienne-Jules Marey $\mathbf{1 6}$ en tant qu'instrument d'analyse du mouvement à la domination des jeux de tir dans les représentations "pixellisées" contemporaines, il y a sans doute là une filiation qui traverse différents dispositifs et pratiques (l'iconographie westernienne, les attractions foraines, les simulateurs de vol pour pilotes d'avions de chasse, etc.) qu'il serait sans doute intéressant d'appréhender dans une perspective intermédiale, voire gender.

L'emprunt effectué par Van Sant à certains jeux vidéo de combat d'une caméra située juste derrière le personnage (dans des jeux dits «à la troisième personne») ne fait que renforcer l'«identification» à la posture des tueurs en termes d'investissement de l'espace17. Certes, un décalage s'opère toutefois, puisque le jeu vidéo auquel joue Alex est un exemple de shooting game "à la première personne", soit un type de représentation caractérisée par une "caméra» subjective rejetant le protagoniste hors-champ dont on ne voit poindre, au premier plan, que le canon de l'arme; or ce point de vue n'est adopté qu'une seule fois dans le film, précisément dans l'un des inserts prospectifs qui ponctuent la récapitulation par Eric des étapes prévues pour la prise d'assaut de l'école $\mathbf{1 8}$. Alors que les images comprises dans les sept autres inserts de cette séquence sont, dans la suite du film, soit reprises à l'identique et inscrites dans une temporalité plus longue, soit totalement élidées, ce plan-ci est le seul à constituer une variation sur un motif qui sera actualisé différemment, avec un point de vue "à la troisième personne", au moment de la représentation de la tuerie. Il en découle que le tir figuré «à la première personne» est strictement associé à l'imaginaire des tueurs, le statut d'insert provoquant une subjectivisation par le montage qui surdétermine - et, en l'occurrence, renforce - celle impliquée par le cadrage. Cet apax en termes de figuration du regard de l'assassin participe du discours relatif à l'influence néfaste des jeux vidéo sur les comportements des adolescents 19 et souligne la volonté de Gus Van Sant de prendre une distance, d'opérer un décentrement par rapport à ce qu'il considère comme étant le "point de vue» emblématique octroyé aux gamers par le dispositif vidéoludique. En outre, il faut noter que la finalité interactive qui caractérise la représentation offerte par ce dernier est inappropriée au cinéma : certaines pratiques peuvent engager des implications fort diffé- 
rentes lorsqu'elles sont transférées dans un dispositif autre 20. Aussi, un jeu vidéo qui aurait été joué et dont le déroulement serait ensuite projeté sur l'écran d'une salle de cinéma serait probablement perçu en termes de construction du point de vue selon les codes cinématographiques, du moins partiellement 21 .

En dépit des conséquences résultant du transfert intermédial, l'adhésion au point de vue des tueurs demeure à notre sens "éthiquement» problématique dans Elephant en ce qu'elle reste déterminée par son emploi dans des jeux ayant pour seul objectif d'immerger le joueur/spectateur dans la mise en scène constamment réitérée d'une situation de massacre. Le cinéaste en a lui-même conscience lorsqu'il déclare dans un entretien s'être laissé "prendre au piège» de l'attraction d'un jeu vidéo, et qu'il constate que l'esthétique pour laquelle il a optée tend à rejouer celle d'un mode de représentation qu'il considère comme l'une des «sources» du massacre:

"Quand j’ai commencé à travailler sur le film, juste après Columbine [...], je ne savais pas ce qu'était un jeu vidéo, je n'y avais jamais joué... J’avais touché aux plus anciens, à la fin des années 1970, mais je n'avais jamais joué sur un récent; j’en ai donc trouvé un appelé Tomb Raider, et à force d'y jouer, je me suis laisser piéger, je suis devenu un accro, au point que lors du tournage du film qui a précédé Elephant, Gerry, mon intérêt portait sur la manière de fonctionner de la caméra dans ce jeu, la manière de suivre les personnages plus que de raconter une histoire. Il ne s'agissait pas d'imiter un jeu vidéo, mais plutôt d'observer comment le jeu s'était immiscé dans ma façon d'envisager le fonctionnement du cinéma. [...] J'ai fait un film qui en quelque sorte a renvoyé comme en écho la tuerie à sa source originale, sans que cela ait été prévu.»22

Même si Elephant n'imite pas littéralement le fonctionnement d'un jeu vidéo, on pourrait toutefois l'envisager comme une production vidéoludique (de très mauvais goût) qui reconstituerait le drame de Columbine en s'inspirant des images prises par les caméras de surveillance et véhiculées par les mass medias - ce principe a d'ailleurs été exploité, de façon «littérale», par Daniel Ledonne, concepteur d'un jeu dans lequel l'utilisateur occupe la place des tueurs lors de la tuerie de Columbine 23 . En effet, le film de Van Sant tend à l'infra-narratif - ou à une forme de narrativité qui procède pour l'essentiel de la configuration spatiale ellemême $\mathbf{2 4}$-, se présente comme un environnement nettement circonscrit et met à disposition du spectateur un certain nombre «d'avatars » choisis tour à tour. D'ailleurs, le cinéaste est allé jusqu'à concevoir pour Elephant un jeu vidéo qui prend pour décor et protagoniste le désert westernien
20 La distinction posée par Gilles Delavaud à propos des effets de la caméra subjective entre le cinéma et la télévision vaut tout autant dans le cadre d'une comparaison entre le cinéma et les jeux vidéo (voir Gilles Delavaud, "Le dispositif télévision. Discours critique et pratique de création dans les années 1940 et 1950 ", dans Mireille Berton et Anne-Katrin Weber, Du téléphonoscope à YouTube. Pour une archéologie de l'audiovision, Lausanne, Antipodes, 2009, pp. 229-248).

21 A moins, bien sûr, que le spectateur ne soit invité à s'y projeter en tant que joueur, comme cela est le cas lorsque des gamers particulièrement chevronnés enregistrent leurs parties et les mettent ensuite à disposition en ligne, accompagnées de leur propre commentaire en voix over, sous une forme proche des matches de sport retransmis à la télévision.

22 Entretien filmé disponible sur le CD-Rom $A$ propos de Elephant, un film de Gus Van Sant, CRDP de l'académie de Nice, 2003 (nous citons ici le texte de la traduction proposée en voix over dans ce document audiovisuel).

23 II s'agit de Super Columbine Massacre RPG (Daniel Ledonne, 2005).

24 A propos des nouveaux liens instaurés par les jeux vidéo entre espace et récit, voir Henry Jenkins, "Game Design as Narrative Architecture", dans Noah Wardrip-Fruin, Pat Harrigan (éd.), First Person. New Media as Story, Performance and Game, Cambridge, MIT Press, 2004, pp. 118-130. 

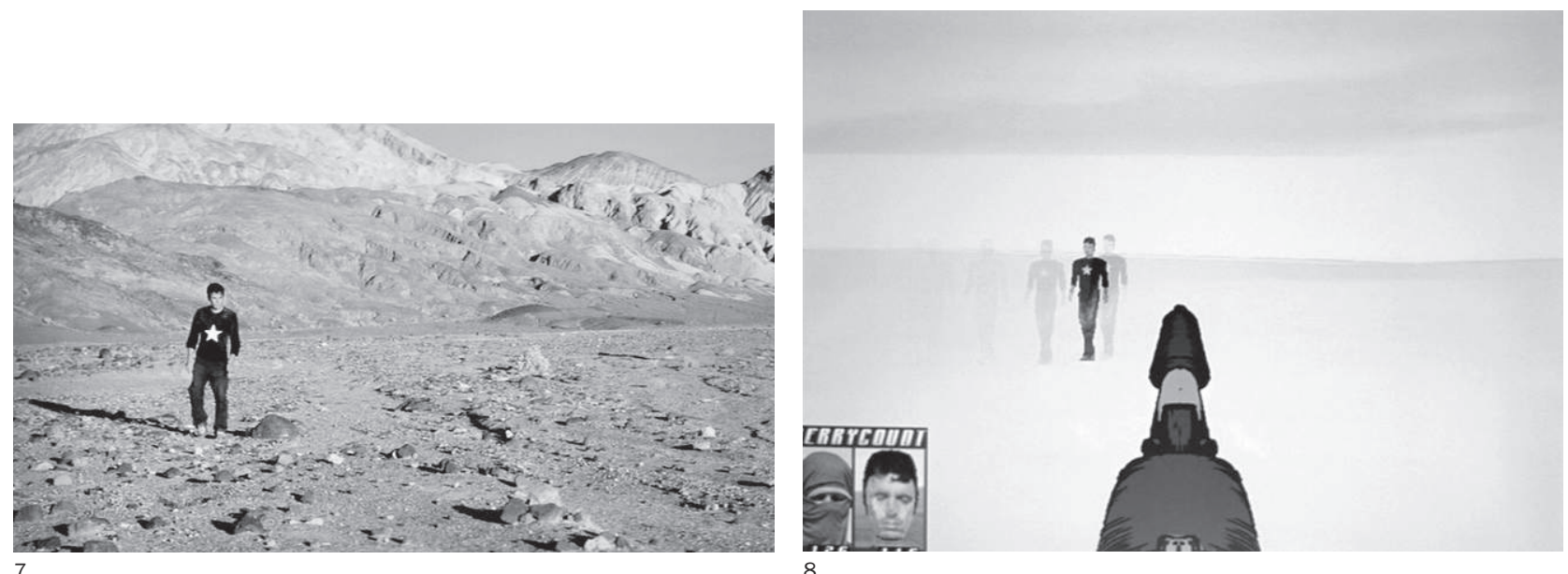

et le (double) personnage éponyme de son film précédent, Gerry. Si l'élaboration de cette création vidéoludique s'explique sans doute par des raisons pratiques (les droits de citation d'un jeu comme Doom auraient sans doute été trop onéreux), il n'en reste pas moins que Van Sant, adepte $\mathrm{du}$ remake sous toutes ces formes (ainsi qu'en témoigne sa reprise plan à plan de Psycho d'Hitchcock), procède à une autocitation qui conduit à une transposition effective d'un film en jeu vidéo de combat: dans les inserts plein cadre sur l'écran d'ordinateur manipulé par Eric dans la chambre de son ami (espace de repli qui constitue la "matrice» du drame), la victime des coups de feu tirés est invariablement le personnage interprété dans Gerry par Casey Affleck, que l'on reconnaît à son T-shirt noir comportant un motif étoilé (fig. 7-8); or ce Gerry-là est

25 Bien que mettant en scène seulement deux personnages, le film Gerry s'organise et se clôt en fonction d'une logique d'apparition/disparition de l'un des protagonistes (voir infra, note 44). Précisons que l'image mentionnée ici (fig. 7) a pour contrechamp un gros plan sur le visage de l'autre Gerry, enturbanné dans une pièce de vêtement bleue (voir l'article de Charles-Antoine Courcoux, fig. 21); l'icône du "terroriste" située au bas de l'écran (fig. 8) renvoie, dans la version vidéoludique de Gerry intégrée à Elephant, à ce personnage, qui, à ce moment-là, "tue du regard" son alter ego dont il commence à ressentir la faiblesse comme un poids. Dans le dispositif interactif du jeu, c'est le regard du gamer qui se substitue à celui, diégétique, de Matt Damon dans Gerry.

26 Olivier Joyard et Jean-Marc Lalanne, op. cit., p. 28. "assassiné » également dans le film, du moins symboliquement ${ }^{25}$. Cette «réécriture» vidéoludique d'un film à tendance expérimentale est certes ironique, mais elle n'en dénote pas moins une attirance pour ce type de représentations. Dans son ensemble, Elephant pourrait même être vu comme une adaptation vidéoludique (factice) d'un film hollywoodien standard puisque, ainsi que l'explique Van Sant, la source sur laquelle il se base est elle-même parente d'une sorte de «film»:

"Tout est parti du massacre dans le petit lycée américain de Columbine. La couverture médiatique a été énorme. J'avais l'impression de regarder un film d'exploitation, tant les images télé et les commentaires étaient dramatisés. $\mathbf{2 6}$

A cet égard, Elephant constitue une déclinaison, arty et "multimédia", d'un film à la facture traditionnelle virtuellement présent à travers le «devenir-images» du drame de Columbine. Cependant, en convoquant 
des motifs prévus pour un dispositif interactif, Gus Van Sant semble vouloir maximiser l'immersion dans le monde qu'il nous donne à voir et à entendre, comme s'il s'agissait d'accéder au hors-champ fantasmatique des images prises par les quatre caméras de surveillance lors du massacre de Littleton ${ }^{\mathbf{2 7}}$, ou plutôt d'instituer la caméra du film elle-même en instance sinon de surveillance, du moins d'observation. Sorte de garde rapprochée, la caméra d'Elephant "prend en chasse» les personnages tandis que le montage tend à signifier que les tenants et aboutissants du drame échappent à toute entreprise de "compréhension".

Il faut toutefois préciser que l'emprunt aux jeux «à la troisième personne" implique que les déplacements de caméra se désolidarisent des personnages, puisque nous ne voyons pas à proprement parler à travers leurs yeux. Cette autonomisation par rapport aux instances diégétiques est particulièrement manifeste lorsqu'un travelling latéral se met en place qui instaure un rapport non plus de proximité, mais de frontalité aux personnages. Ainsi, dans les séquences où les lycéennes choisissent un repas à la cafétéria, la caméra passe de l'autre côté du comptoir, et, dans un mouvement de droite à gauche amorcé par les protagonistes mais amplifié (comme s'il s'agissait, peut-on en penser dans un premier temps, de suivre une figurante qui a surgi au premier plan), elle rejette Brittany, Jordan et Nicole hors-champ pour sonder brièvement les «coulisses", du côté des cuisines (fig. 9-12); il en va de même au guichet du secrétariat, où la caméra quitte les adolescents dont elle suivait le déplacement - également de droite à gauche, indice d'un sens de «lecture» bouleversé - pour pivoter et découvrir, dans la pièce adjacente sur laquelle s'ouvre une porte, une réunion d'employés de l'établissement qui, de toute évidence, s'apprêtent à fêter un événement dont nous ne saurons rien. Cette séquence s'interrompt abruptement, sanctionnant le dépassement des limites que le cinéaste semble s'être fixées en termes de récit (l'accueil du personnage - qui n'est pas un élève du lycée - par l'exclamation enjouée «Surprise! » demeure une énigme, un possible narratif non actualisé), de genre - il ne s'agit pas pour Van Sant de réaliser un documentaire sur une institution comme a pu le faire Frederick Wiseman dans High School (USA, 1968) - et, surtout, d'organisation spatiale.

Rarement en effet nous n'avons vu au cinéma - exception faite du cinéma expérimental, dont Elephant hérite de certains traits en les adaptant habilement à un cinéma d'auteur qui se veut "indépendant» - un tel souci de délimiter l'espace (de la fiction), qu'il s'agisse du respect de frontières diégétiques ou du travail sur les limites du cadre. Sans doute symptomatique d'une conception "spatialisante" de la représentation qui s'est généralisée avec les usages récents des nouvelles technologies $\mathbf{2 8}$,
27 Notons que ces images de caméras de surveillance sont citées dans le film de Michael Moore, simultanément aux enregistrements sonores de messages de détresse des élèves menacés et de leurs parents inquiets, dans une séquence de montage d'environ deux minutes qui alterne images plein cadre et écrans quadripartites.

28 Voir à ce propos Lev Manovich, The Language of New Media, Cambridge/London, MIT Press, 2001. La réflexion de Manovich sur la "spatialisation" du montage porte certes sur un cinéma qui, recourant massivement aux effets numériques, peut sembler aux antipodes de l'entreprise de Gus Van Sant. Cependant, comme on le constate avec l'emprunt aux jeux vidéo, Elephant participe en partie de ce contexte médiatique. 

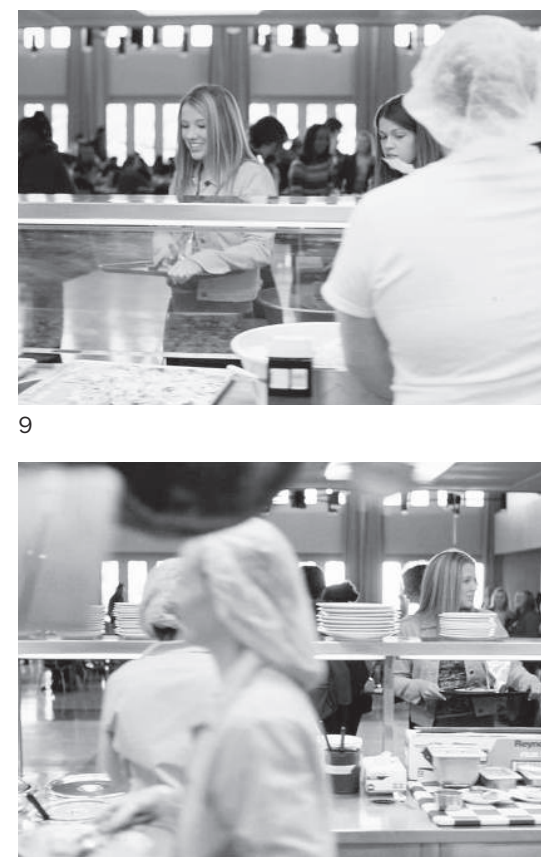

11

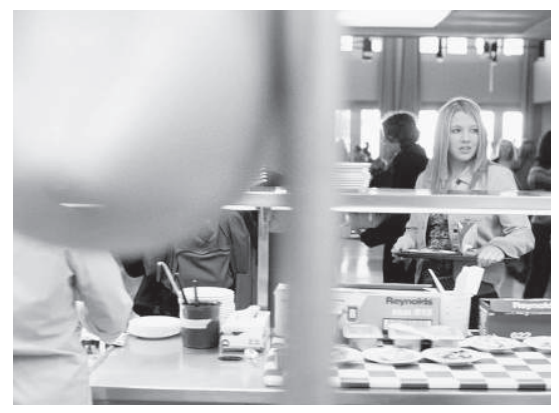

10

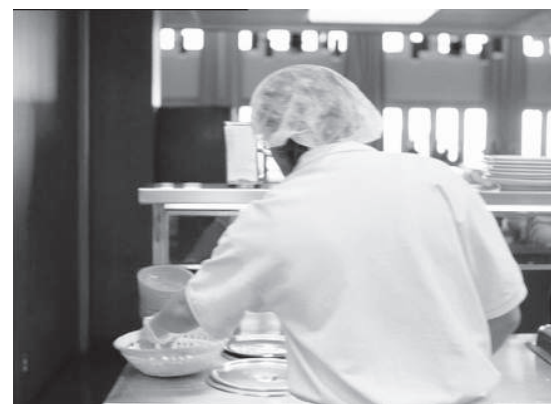

12

cette tendance à faire primer l'espace sur l'action, le lieu sur le personnage, le descriptif sur le narratif, mérite une analyse de détail que nous proposons de mener à partir d'une séquence qui est à cet égard emblématique, située relativement au début du film et encadrée par deux plans quasi identiques du proviseur toisant avec méfiance un élève, John, situé hors-champ (fig. 13-25). Ce retour d'une même image (dont seule l'échelle scalaire varie) indique, au niveau de la temporalité, que tout ce qui a été montré dans la séquence que nous analyserons s'est déroulé simultanément à ce qui précédait l'arrivée au secrétariat de John, le premier personnage que nous avons accompagné dans ses déplacements $\mathbf{2 9}$

29 Notons que cette place liminaire confère à John un statut privilégié (confirmé par le matériel promotionnel du film, où ce jeune homme blond au T-Shirt jaune est omniprésent), celui de nous faire entrer dans le film (voir à ce propos l'article de Charles-Antoine Courcoux dans le présent dossier). Les tueurs semblent en quelque sorte tenir compte de ce statut lorsqu'ils lui conseillent de ne plus revenir dans l'établissement ("Casse-toi et ne reviens pas - ça va chier!"), tandis que c'est autour de John que se noue l'unique effet de clôture du film, scellé autour d'un rapprochement affectif entre - action décisive, puisqu'il rencontre sur son trajet les tueurs qui s'apprêtent à pénétrer, armés, dans le bâtiment. Par rapport à la piste narrative de ce personnage, la séquence dont nous traiterons, dédiée à partir de la fin du premier plan à Nathan (qui rejoint sa copine Carrie), semble plus périphérique: elle fait office de parenthèse avant le retour à John assis au secrétariat, soit dans l'un de ces lieux situés à la croisée des chemins, là où s'opèrent les bifurcations du récit et où la violence peut potentiellement éclater. 


\section{Le marquage (énonciatif) du terrain de jeu}

La séquence en question débute par un long plan au cadrage obstinément fixe (en dépit d'un léger bouger de l'appareil de prise de vues qui témoigne de la présence du filmeur), comme si nous étions face à un moniteur d'une caméra de surveillance : les actions se déploient aléatoirement, indépendamment de l'acte de filmer et, souvent, dans l'espace hors-champ (fig. 14-24). Il s'agit d'un terrain de jeu sur lequel, au premier plan de l'image, des adolescents s'entraînent au football: les corps s'esquivent, s'agrippent, se heurtent, dans une agitation apparemment soustraite à toute règle, le spectateur étant incapable de comprendre quelque chose à la partie (pour autant qu'il y en ait une) ${ }^{\mathbf{3 0}}$. Le caractère pluricentré de l'image rappelle les vues, elles aussi fixes ${ }^{31}$, du cinéma des premiers temps. L'occultation de tout ce qui excède la délimitation spatiale imposée à l'image - alors même que les joueurs sortent constamment du champ, comme un appel à les suivre qui demeure entravé (d'autant plus qu'Elephant obéit jusque-là au principe de la filature) - souligne la présence de la caméra, l'origine d'un regard assigné à un espace donné qui, en une légère plongée, enregistre "mécaniquement» le déroulement de l'action (car on comprend bien qu'il ne s'agit pas là du regard d'un personnage). Le hors-champ dont l'absence est exhibée ne concerne pas seulement le bord droit du cadre où les joueurs disparaissent puis d'où ils resurgissent, mais également ce que Noël Burch a appelé, dans sa typologie des espaces hors-champ, le "cinquième segment", c'est-à-dire le pan de "mur» correspondant à l'endroit où se situe la caméra ${ }^{32}$. En effet, l'un des joueurs s'élance à l'avant-plan pour se jeter sur le ballon (fig. 16), tandis que, plus tard, Nathan s'y penche pour saisir son sweatshirt (fig. 20). Certes, la caméra reste indemne, intouchée par ces personnages qui se précipitent dans sa direction; néanmoins, la place qu'elle occupe est ostensiblement signifiée dans le champ lui-même par de tels débordements dans un en deçà du champ dont le cinéma traditionnel prend soin de gommer toute trace. Ici, l'œil sous lequel se déroulent les actions affiche sa présence. Dans les termes proposés par les théories de l'énonciation au cinéma qui tentent de rendre compte de la façon dont un film est susceptible de rompre la transparence de l'histoire pour s'afficher en tant que discours ${ }^{\mathbf{3} 3}$, on peut dire que l'ouverture de cette séquence d'Elephant, à notre sens emblématique de l'ensemble du film, se veut marquée au niveau de la monstration $\mathbf{3 4}$ : le spectateur est invité à percevoir qu'une instance lui donne à voir le représenté, et non que le monde se donne à lui sans médiation aucune.

Par conséquent, le filmage affiche une indifférence à la vie des personnages, qui, présentée de façon fragmentaire, nous apparaît dès lors
30 On le sait, la retransmission télévisuelle de matches de football est précisément caractérisée par une multiplication des angles de prise de vues, par une mobilité du cadre et par une orientation de l'attention du spectateur sur un élément, le ballon (qui, ici, sort constamment du champ). On pourrait dire à ce titre que ce plan d'Elephant radicalise, en délaissant même ses personnages qu'il perd dans un plan d'ensemble, la posture pour laquelle optent Douglas Gordon et Philippe Parreno dans Zidane. Un portrait du $21^{\text {ème }}$ siècle (France, 2005) (voir à propos de ce film l'article de Claus Gunti, "La réalité remise en question: le statut de l'image dans l'œuvre de Philippe Parreno", Décadrages, $n^{\circ} 13$, automne 2008, pp. 37-40).

31 Lorsque les vues Lumière sont filmées avec un appareil mobile, il s'agit, comme dans d'autres séquences d'Elephant, d'un travelling avant "naturalisé" par un déplacement diégétique (en train, en gondole, etc.).

32 Noël Burch, Une praxis du cinéma, Paris, Gallimard, 1986 [1967], p. 39.

33 Notions proposées dans le champ de la linguistique par Emile Benveniste ("Les relations de temps dans le verbe français", dans Problèmes de linguistique générale, vol. 1, Paris, Gallimard, 1959), reprise - de façon plus strictement oppositionnelle - par Christian Metz (Le signifiant imaginaire, Paris, Christian Bourgois, 1977). Voir à ce propos notre ouvrage La fiction au cinéma, Paris, L'Harmattan, 2001, chapitre 3.

34 Nous empruntons la notion de "monstration" à André Gaudreault (Du littéraire au filmique, Québec/Paris, Nota Bene/Armand Colin, 1999 [1988]), dont la conceptualisation de l'énonciation narrative présente l'intérêt de dissocier le niveau de la mise en cadre associée au tournage - celui de la monstration - de ce qui touche à l'organisation discursive effectuée au niveau du montage (la narration proprement dite). Pensé pour le cinéma des premiers temps, il n'est pas surprenant que ce modèle s'avère pertinent à l'analyse d'Elephant, dont nous avons souligné certaines parentés avec le cinéma des débuts. 
d'autant plus «réelle». Au cours de ce plan, le personnage de Michelle, dont on sait la gêne qu'elle éprouve à faire du sport avec ses camarades, à être "l'objet des regards", vient momentanément s'inscrire dans le champ, seule, au premier plan et au centre de l'image (fig. 18): dissimulant en partie les joueurs situés derrière elle qui deviennent dès lors secondaires, la jeune fille s'interrompt dans sa course pour regarder le ciel, dans un moment de rêverie souligné par un léger ralenti, comme s'il s'agissait pour Gus Van Sant de faire durer quelque peu cette part d'insaisissable (voire d'irrémédiable) inscrite en certains instants. Pratique récurrente dans le film, le ralenti tend à particulariser certains moments
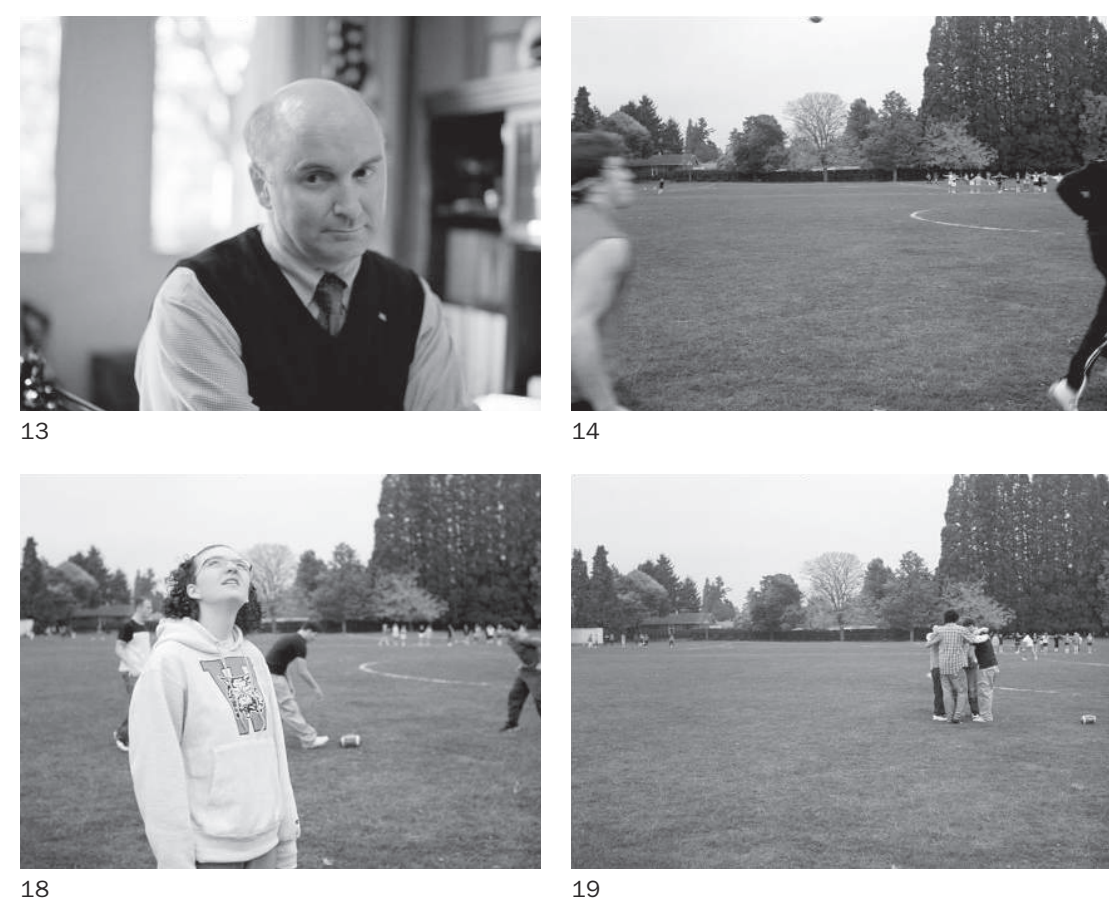

18

19
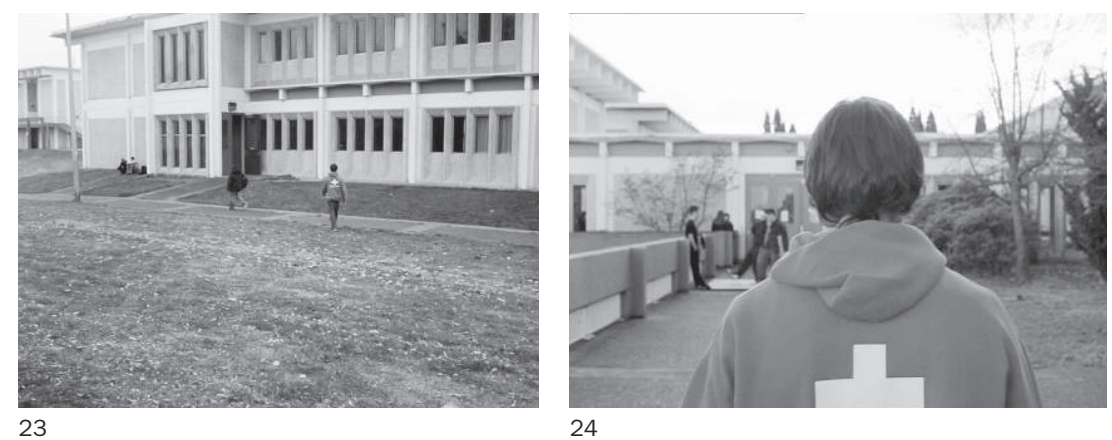
et certaines attitudes des personnages. Le plus marquant "consacre» l'instant où John joue avec le chien de l'un de ses camarades, alors que les tueurs s'approchent de lui à l'arrière-plan, traversant la pelouse. La variation de vitesse du défilement des images suggère l'imminence du drame, comme lorsque le ciel se couvre avant l'orage - motif qui correspond à ce que Michelle est probablement en train d'observer durant la ralenti de la séquence examinée ici. Les plans sur les nuages en mouvement sont quant à eux filmés en accéléré, ce qui provoque une même étrangeté que les ralentis sur les personnages, mais d'une manière qui semble affranchie de toute contrainte "terrestre». L'irréalisation momentanée

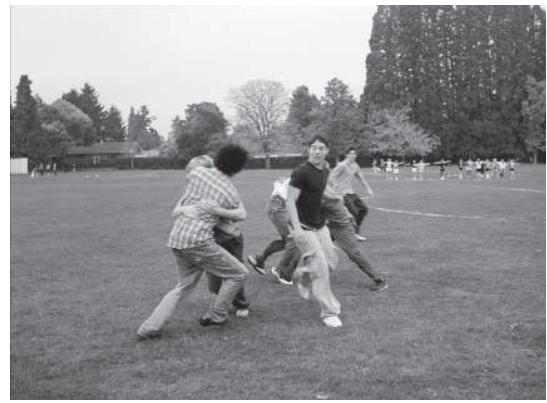

15

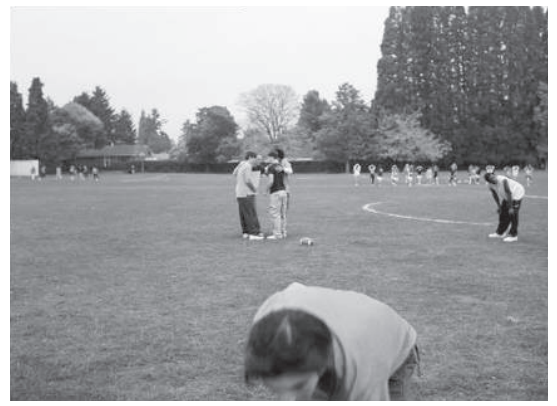

20

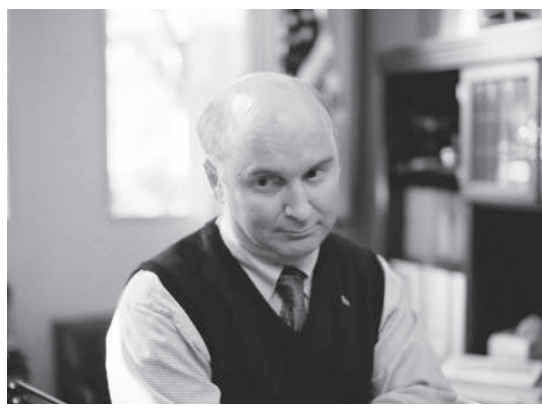

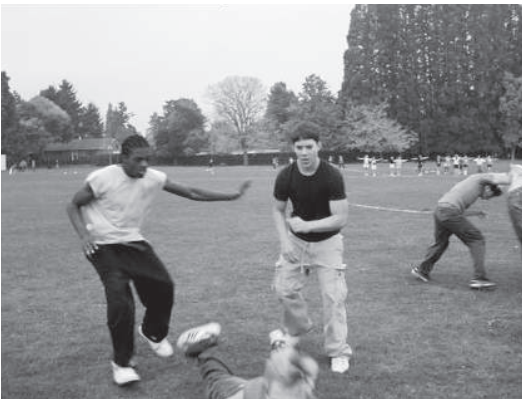

16

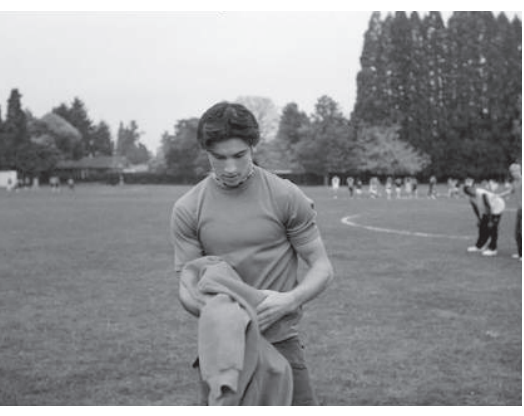

21

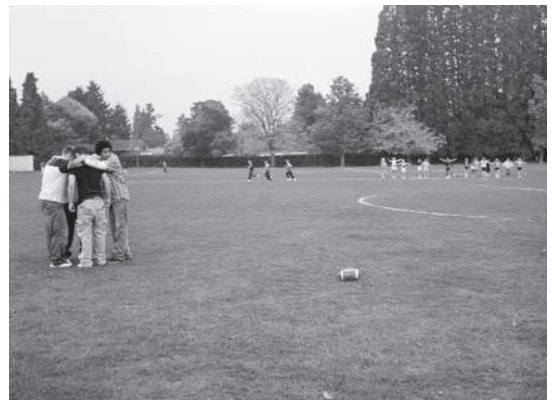

17

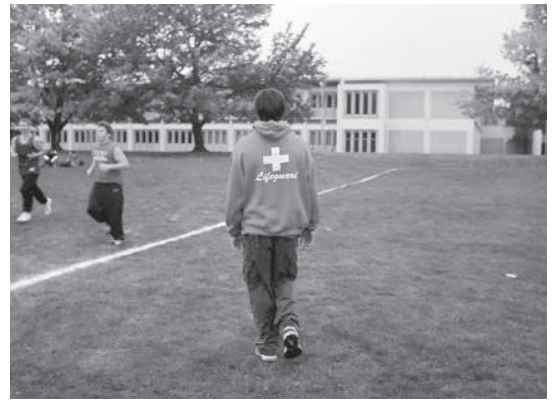

22 
35 A propos du motif des nuages dans l'œuvre du cinéaste, voir Edouard Arnoldy, op. cit., en particulier pp. 25-33.

36 Eric explique à son complice: "Quand tu arrives à la ligne jaune, tu passes au plan B. [...] Moi j'arrive à la ligne rouge, le hall, avec les plus belles cibles, les sportifs." Le schéma qu'il consulte ressemble fortement à un plan d'évacuation en cas d'incendie sur lequel différents trajets seraient indiqués; nous reviendrons sur la question des couleurs dans le film. de la représentation qui résulte du ralenti se fait l'expression d'une fascination du cinéaste pour la captation même du monde dans ce qu'il recèle de fugitif, et tend à subjectiviser passagèrement l'image de l'intérieur, indépendamment du lieu d'où l'on regarde: en effet, cette singulière "respiration" de l'image est mise au bénéfice du ressenti de la joggeuse essoufflée, qui semble s'extraire, l'espace d'un instant - dans le sens où il s'agit d'un intervalle court, mais aussi, littéralement, d'un instant ancré dans un espace donné -, du malaise dont elle souffre. En gagnant le personnage comme le spectateur, la stase contemplative est l'un des procédés permettant au cinéaste de concilier l'asymétrie des regards - nous n'épousons pas celui de Michelle - et l'empathie perceptive (comme elle, nous avons «la tête dans les nuages", ces derniers étant visualisés ailleurs dans le film) ${ }^{35}$. Cette brèche dans le flux du quotidien a néanmoins tôt fait de se résorber pour laisser la place à la concrétude des corps et à l'inéluctable déploiement de mouvements énergiques (et virils).

La contemplation, en elle-même, postule l'exhibition d'un sujet du regard. Mais quel est ce lieu où «l'œil d'Elephant» semble s'être fixé, le temps de ce plan? Pour saisir les implications d'un tel ancrage, il est nécessaire d'examiner la dynamique des entrées et sorties de champ, et l'inscription des actions dans la profondeur du champ (dont l'appréhension est favorisée sur le plan optique par l'utilisation d'une grande profondeur de champ). Au tout début du plan, trois coureurs passent en trombe devant la caméra, l'un chassant l'autre hors du champ (fig. 14), silhouettes floues au premier plan de l'image dont l'apparition successive introduit l'idée d'une cadence des entrées dans le champ, d'un jeu rythmique sur le vide et le plein. Or, lorsqu'ils passent à l'arrière-plan, ces coureurs suivent exactement la ligne blanche dessinée sur le gazon par une traînée de sable: on les voit subrepticement sur le bord droit du cadre - précisément là où Michelle poursuivra sa course, dont la trajectoire est également calquée sur cette ligne, avant de rejoindre le groupe de jeunes filles (fig. 19) -, puis à l'arrière-plan, suivant une trajectoire exactement parallèle au bord inférieur du cadre (fig. 17). Le trajet effectué par ces joggers respecte donc scrupuleusement la délimitation du terrain de football : à l'instar des concepteurs de plans d'aménagement semblables à celui dont se servent les tueurs pour planifier et optimiser la tuerie ${ }^{36}$, le cinéaste organise l'espace dans le "plan " (qui tient autant de l'unité filmique que du schéma d'architecte) en fonction des frontières que lui offre l'espace réel. La caméra, elle aussi, se trouve exactement sur la ligne, cette dernière étant remplacée par le bord même du cadre : le terrain de jeu est également celui de la fiction. Lorsque Nathan 
dépasse la ligne (et donc sort du cadre) pour ramasser son sweat-shirt, il préfigure la suspension de la logique centrifuge de l'image qui s'effectuera peu après, lorsqu'il sortira complètement du champ pour quitter le terrain. Dès lors, la caméra se met en mouvement pour le suivre. Etonnamment, cette rupture de la fixité du cadre n'occasionne aucune coupe, l'organisation filmique continuant de se jouer au strict niveau de la monstration, dans un plan unique dont la durée totale est de six minutes (si l'on considère que la première coupe intervient avec l'insert de la mention «Nathan \& Carrie»).

Il nous paraît symptomatique que ce plan, dont nous avons relevé les caractéristiques et les implications en termes de marquage énonciatif, embraie sur l'une des filatures où la caméra demeure située dans le dos du protagoniste (fig. 22-24). Car nous sommes bien là dans un cas passablement différent de ce que François Jost a proposé de nommer "l'ocularisation interne primaire», soit l'assimilation exacte de l'œilcaméra au regard du personnage 37 : le spectateur voit la même chose que ce dernier (mais, comme nous l'avons dit, pas tout à fait de la même manière) puisqu'il marche dans ses pas, mais il ne cesse, simultanément, de voir par ailleurs ce personnage même. Toutefois, la visualisation du personnage n'a qu'une très faible incidence en termes de focalisation puisque son visage, qui constitue le siège de l'identification (des affects), nous demeure caché38. Si certains plans dans lesquels la caméra est située très près de la tête du personnage (par exemple fig. 24) peuvent laisser penser que ce dernier apparaît en amorce (en vertu de ce que certains appellent une caméra "semi-subjective»), d'autres creusent la distance, comme cela est significativement le cas dans la seconde partie du plan dont il est question ici: la caméra se met certes en branle pour suivre Nathan (fig. 22) comme elle l'a fait pour John et Elias, mais elle semble affectée d'une sorte de pesanteur, comme si elle avait été engourdie à force d'avoir été rivée sur la même portion d'espace. Elle ne rejoint en effet pas Nathan qui s'éloigne, s'arrêtant à distance de ce dernier (fig. 23) tandis qu'il s'apprête à pénétrer dans le bâtiment par le franchissement d'un seuil auquel répond la frontière que s'est fixée la caméra en s'immobilisant. Même si, dans la suite du trajet parcouru par le jeune homme à l'intérieur et à l'extérieur du bâtiment, la caméra se montre tout à fait solidaire de son déplacement en conservant une distance faible et constante, ce plan-ci suggère qu'il n'y a pas nécessairement dans Elephant interdépendanceentre les mouvements d'appareil et la marche des protagonistes. Instance libérée des contraintes diégétiques, la caméra exhibe sa présence, et porte un certain regard, presque entomologique, sur ces «insectes» qui s'agitent jusqu’à se donner la
37 François Jost, L'œil-caméra. Entre film et roman, Lyon, PUL, 1987, pp. 23-28.

$38 \mathrm{~A}$ propos de la question de la "caméra subjective", de la théorisation à laquelle elle a donné lieu et de ses liens (non nécessaires) avec la "focalisation interne" au sens de la narratologie genettienne, voir l'article de Valentine Robert, "Le scaphandre et le papillon et l'adaptation filmique du 'je, littéraire: I'œil qui écrit", Décadrages, n 16-17, pp. 104-117; l'auteure revient notamment sur l'exemple du film Lady in the Lake (La Dame du lac, Robert Montgomery, 1947), cas d'école lorsqu'il s'agit de montrer que l'usage systématique de la caméra subjective a un effet plus exhibitionniste et réflexif qu'immersif. Gilles Delavaud a de son côté montré qu'il en allait différemment dans le dispositif télévisuel, duquel, sans nul doute, Elephant s'inspire également dans sa pratique de la filature, devenue un lieu commun d'un certain type de reportage (voir Gilles Delavaud, op. cit.). 
mort. Alors que le montage permet de construire, sur le plan de la narration, une structure polyphonique qui invite le spectateur à passer d'un personnage "focal» à l'autre (sans toutefois ne jamais briser le rapport d'extériorité qui est instauré avec chacun d'eux), l'affirmation systématique de la présence de la caméra et de choix marqués au niveau du filmage produit, sur le plan de la monstration, un fort effet «monophonique». Bien qu'éclaté dans sa structure, Elephant paraît ainsi fortement homogène dans sa facture, affichant un style qui fait office de signature, ainsi que le remarque Edouard Arnoldy à propos des amples mouvements de caméra:

«[...] ces travellings affichent un détachement certain vis-à-vis des personnages que la caméra croise, perd de vue et, parfois, retrouve au détour d'un couloir ou d'une rue. Van Sant reprend vite la main pour mettre les personnages à une juste distance, comme pour affirmer la place, cardinale, directrice, du cinéaste. » $\mathbf{3 9}$

Dans le champ du cinéma "d'art et essai», une telle auto-désignation par le film de ses propres pratiques de filmage favorise (voire programme) une lecture de type esthétique et auteuriste.

\section{Une "focalisation" sur la matière de l'image}

La gestion du "point de vue» filmique dépend de l'endroit d'où chaque plan est filmé, mais aussi, tout simplement, de ce que nous donne à voir l'ensemble du film. Au hors-champ de l'image qui détermine certaines particularités de la monstration correspond l'éviction complète de portions de récit par le montage. A ce titre, Elephant se veut très elliptique et, à l'instar d'autres films du cinéaste (Gerry, Last Days,...), n'offre pas

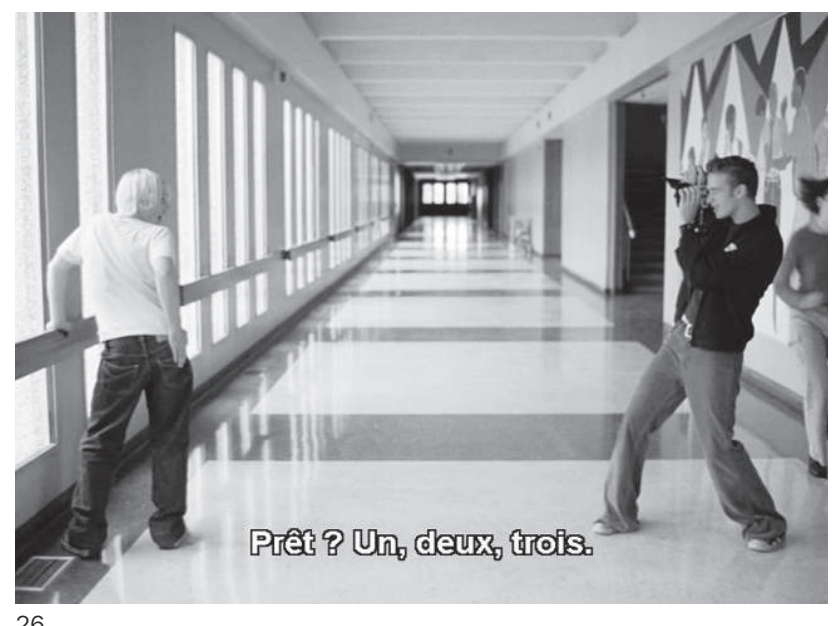


d'exposition permettant de livrer au spectateur certaines informations sur les adolescents à la dérive qui peuplent le film. Notre savoir sur eux se limite aux actions montrées, qui consistent le plus souvent en des déplacements, c'est-à-dire en un type de contenu narratif qui, traditionnellement au cinéma, disparaît dans des ellipses. Quant aux dialogues, fragments de discussions quotidiennes de jeunes gens dans les couloirs de leur école, ils sont rares et superficiels. Avant que les tueurs ne se mettent en route pour le lycée (à environ une heure du début du film), renforçant le statut central de la piste narrative dans laquelle ils s'inscrivent et autour de laquelle s'organisent toutes les autres jusqu'à, désormais, se fondre dans une chronologie unique, les inférences du spectateur sur la diégèse ne portent pas tant sur la construction des personnages que sur la compréhension de l'organisation temporelle. A cet égard, ce ne sont pas les protagonistes en tant que tels qui importent, mais les moments où ils se rencontrent. En effet, la convergence momentanée des pistes narratives permet au spectateur d'établir une relation de simultanéité entre les événements et, ainsi, de se faire progressivement une représentation mentale de l'espace-temps global de la diégèse. Comme une gare de triage, ces scènes-repères ordonnent la bifurcation des pistes en les situant temporellement les unes par rapport aux autres. En de tels instants - dont la représentation se présente parfois sous la forme d'un ralenti (John croisant les tueurs, Nathan passant devant le groupe de filles) -, le temps tel que l'appréhende le spectateur "se fixe»: ainsi n'est-ce pas un hasard si l'une de ces scènes, montrée à trois reprises dans le film (tout d'abord en suivant John, puis Elias, et enfin Michelle, qui traverse subrepticement le champ en longeant
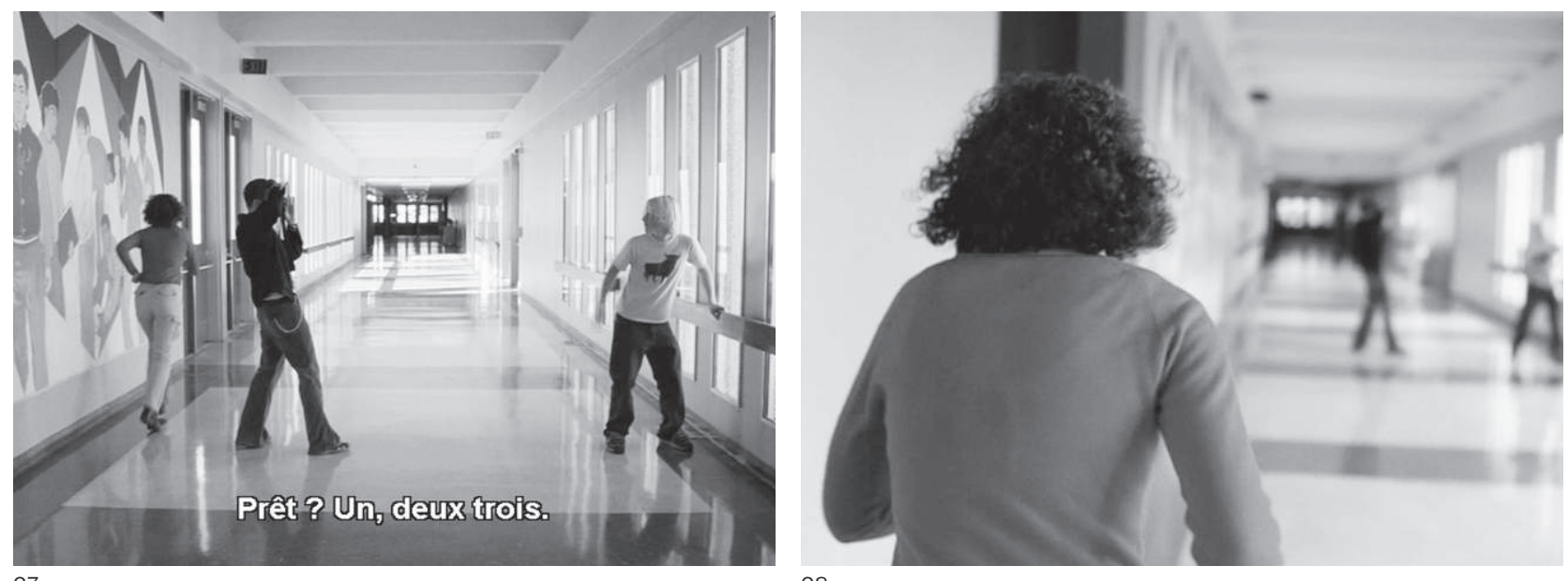
40 Remarquons que Van Sant effectue également ailleurs un travail sur l'image qui se fige (ou, inversement, qui s'anime après la pose): ainsi, dans My Own Private Idaho (USA, 1991), les couvertures des diverses revues érotiques gay disposées à la verticale sur l'étalage d'un kiosque s'animent-elles soudain, les personnages de chacune d'elles conversant entre eux (réactivant un "truc" du cinéma des premiers temps que Méliès avait utilisé dans Les affiches en goguette, France, 1905); voir aussi, dans ce même film de Van Sant, les deux séquences d'étreintes, où les corps nus prennent la pose dans une série de "tableaux vivants" saisis par de brefs plans rapprochés qui, de même que dans certains films de Jean-Luc Godard des années 1960 (notamment Une femme mariée, France, 1964), "épurent" la figuration des relations sexuelles. Van Sant s'amuse également à utiliser un arrêt sur image ironique au début de Even Cowgirls Get the Blues (USA, 1993).

41 On trouve un exemple similaire lors de l'aller-retour en voiture des deux personnages qui viennent rendre visite à Blake dans Last Days (USA, 2005): ce n'est qu'au moment où leur véhicule croise celui des amis du musicien que le spectateur est à même de situer temporellement cette visite, et de comprendre que certaines actions montrées préalablement se passent en fait après le départ des visiteurs éconduits. A l'instar de la séquence d'Elephant évoquée ici, qui se distingue de la précédente par un renversement à $180^{\circ}$ de l'angle de prise de vues, le chemin de campagne est le lieu d'une inversion: la voiture est d'abord filmée à travers le pare-brise, puis, au retour, à travers la vitre arrière; les squatteurs de la villa de Blake roulent en direction de son domicile, alors que nous venons de les voir prendre la décision de le quitter.

42 Film sorti peu avant Elephant dans lequel les flash-backs se voient artificiellement affectés, pour tous les éléments de l'image, d'une couleur unique qui est fonction du narrateur auquel est imputée l'analepse.

43 Rappelons que la police utilise, elle aussi, des couleurs pour identifier les différents acteurs du drame dont la présence est indiquée dans des diagrammes: ainsi les témoins sontils figurés par un point vert, tandis que les blessés sont indiqués en jaune et les personnes décédées en rouge (puis en gris lorsque l'action dont elles ont été victimes est achevée) (voir la référence de la note 14). la paroi, fig. 26-28), est précisément dédiée à la "pose» de John devant Elias qui le photographie $\mathbf{4 0}$. Le pendant cauchemardesque de cette rencontre surviendra plus tard, lorsqu'Elias photographiera l'assassinat de Michelle, avant d'être lui-même victime d'une balle.

De tels instants "prégnants", qui correspondent le plus souvent à des situations où, simplement, deux groupes de protagonistes se croisent41, montrent combien, dans Elephant, un point de vue donné se définit moins en lui-même que dans la relation contrastive qui est instaurée avec d'autres pistes. Même si, comme on l'a dit, un style de filmage identique unifie l'ensemble des pistes, chacune d'elles tend toutefois à se singulariser, ne serait-ce qu'à travers la démarche propre à chaque actrice ou acteur. Chacun des personnages, clairement identifié par son habillement pourvu d'un sigle distinctif (souvent un logo) et d'une couleur dominante immédiatement reconnaissable, imprègne les images consacrées à la piste narrative qui lui est associée. Si Gus Van Sant n'opte pas pour un usage aussi clairement subjectivisant de la couleur que celui de Zang Yimou dans Hero (Chine, 2002) $\mathbf{4 2}$, la séquence de «rencontre» entre les trois personnages que nous avons mentionnée cidessus témoigne de l'attribution à chaque personnage d'une dominante chromatique: l'image tend vers le beige pour les plans appartenant à une séquence «focalisée» sur John, vers le bleu pour Elias et vers le blanc pour Michelle $\mathbf{4 3}$. Eric, le tueur, apparaît quant à lui beaucoup moins "lumineux» que ses camarades. On rétorquera que c'est là un critère subjectif (précisément!), contestable en de nombreux plans en fonction des conditions d'éclairage du moment de la prise de vues. Mais c'est pourtant bien ainsi que Gus Van Sant touche au cœur de l'expression

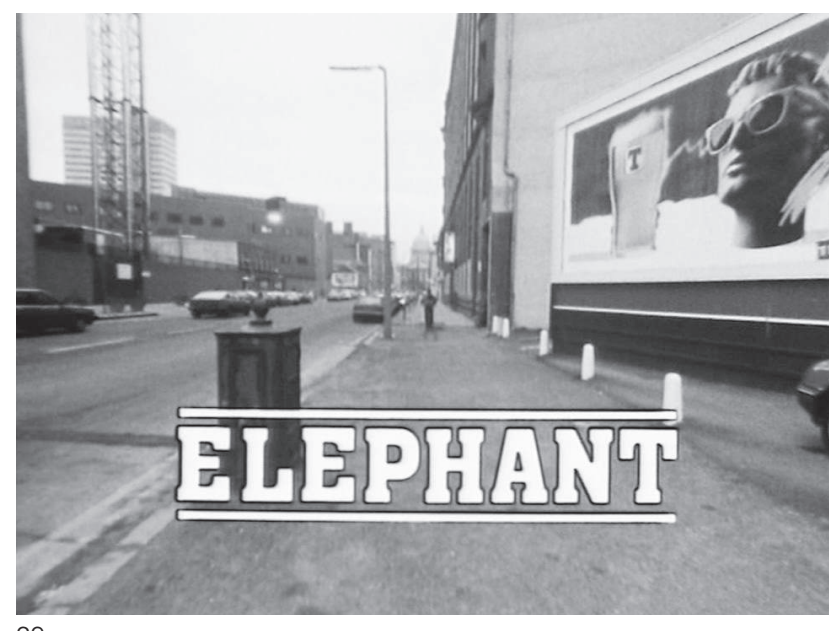


filmique d'un "point de vue» sur le monde: il ne s'agit pas principalement d'un mode d'organisation narratif, mais d'une valence particulière conférée au "grain" de l'image. En alternant, grâce aux variations de mise au point, les contours à la netteté tranchante avec des zones floues qui contaminent l'écran jusqu'à le menacer de devenir une surface abstraite, Van Sant et son caméraman Harris Savides $\mathbf{4 4}$ font du point de vue une question avant tout plastique et sensorielle. Dans Elephant, l'usage du "plan-séquence en profondeur de champ» est anti-bazinien (à l'exception du premier plan de la séquence du terrain de football analysée ci-dessus) dans la mesure où, en raison des nombreux changements de focale et de mise au point, il vise à orienter ostensiblement le regard du spectateur, se substituant au montage; pourtant, c'est justement grâce à cette esthétique que le film touche à «l'ambiguïté du réel» qui était si chère au critique français $\mathbf{4 5}$, et baigne chaque image d'une subjectivité diffuse.

\section{D'un Elephant à l'autre}

Même si la critique a fréquemment relevé la mise à distance des personnages chez Van Sant, son film n'est pas rétif à toute manifestation d'émotions. Il suffit pour s'en convaincre de le comparer au film dont Gus Van Sant s'est inspiré au point d'en emprunter le titre: Elephant d'Alan Clarke (GB, 1989) (fig. 29-35). La froideur clinique de ce court métrage réalisé pour la $\mathrm{BBC}$ tient à la systématicité et à l'austérité avec lesquelles sont juxtaposées les différentes séquences qui le composent, toutes exclusivement consacrées à la représentation de meurtres sordides et totalement gratuits. Dans ce film, chaque nouvelle scène possède son autonomie et nous montre le déplacement d'un personnage dont
44 Savides, qui optera pour une esthétique à certains égards similaire dans Zodiac (David Fincher, USA, 2007), s'était déjà chargé de manier la caméra dans la précédente fiction de Van Sant, Gerry (2002), où les changements de mise au point servaient à subjectiviser la représentation. Ainsi, l'un des deux Gerry (puisque les deux personnages, dont l'un est interprété par Casey Affleck et l'autre par Matt Damon, portent le même prénom et sont dépourvus de "nom propre"), celui qu'incarne Affleck, filmé en mouvement et sans coupe devant son camarade avec une faible profondeur de champ, devient-il flou alors que la mise au point s'effectue progressivement sur le visage de Damon situé à l'arrière-plan: l'idée qu'un personnage relaie l'autre, voire que les deux ne font qu'un (l'un des deux pouvant être vu comme une projection fantasmatique, "subjective", de l'autre) passe par un travail effectué au moment même de la prise de vues.

45 André Bazin, "L'évolution du langage cinématographique" [1950-1955], dans Qu'est-ce que le cinéma, Paris, Cerf, 1994 [1958], en particulier pp. 75-76.

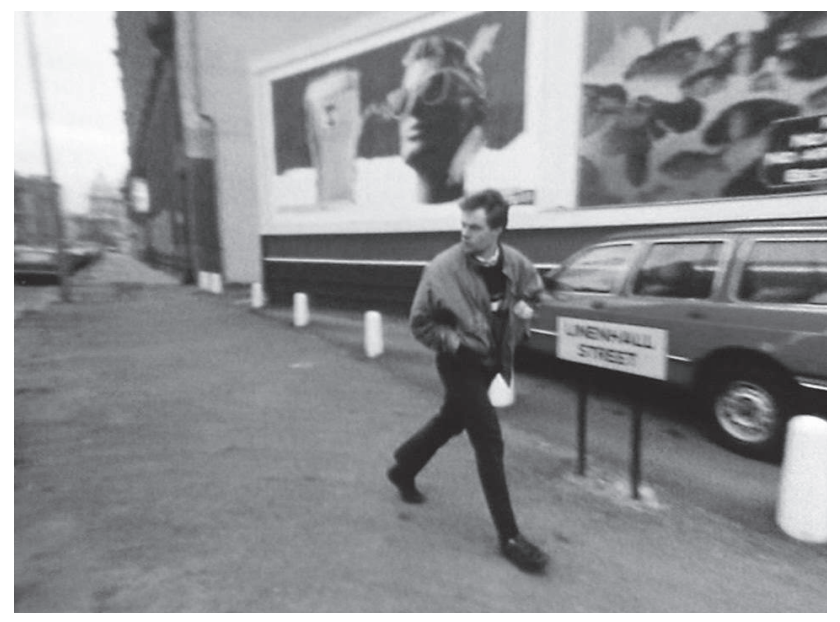

30

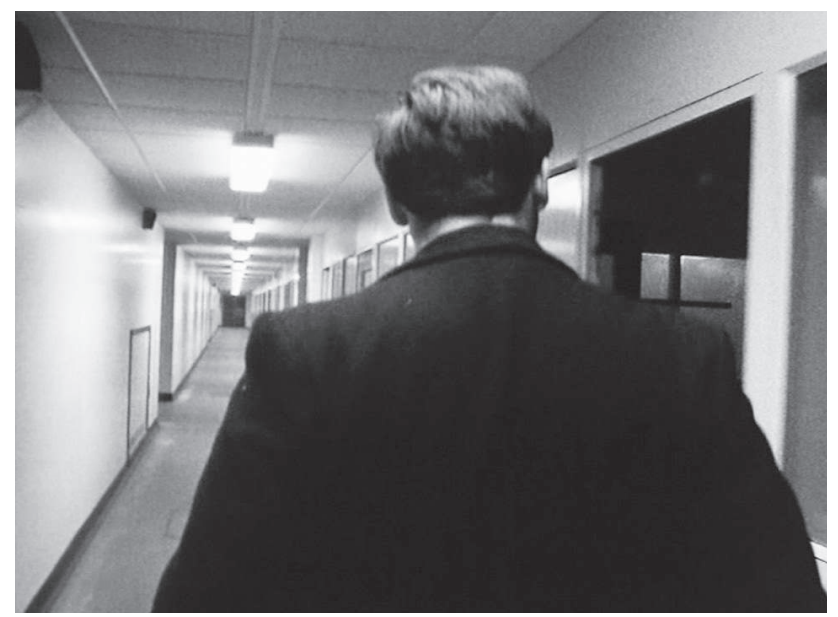

31 


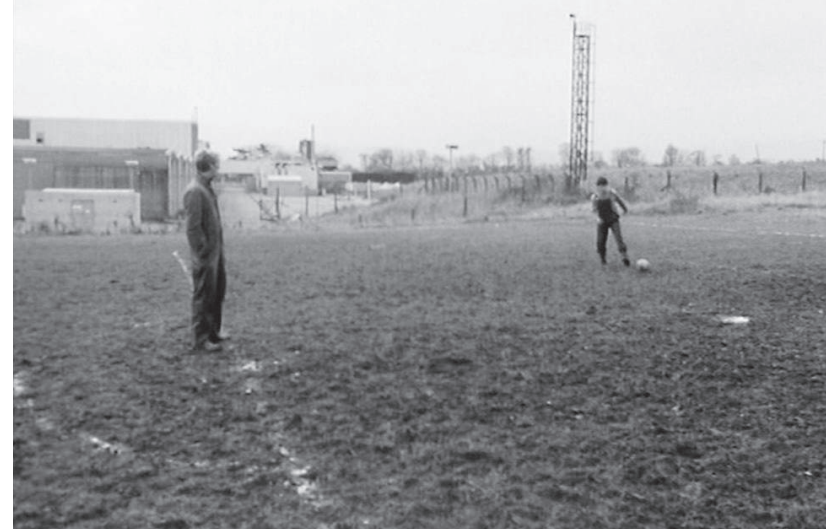

32

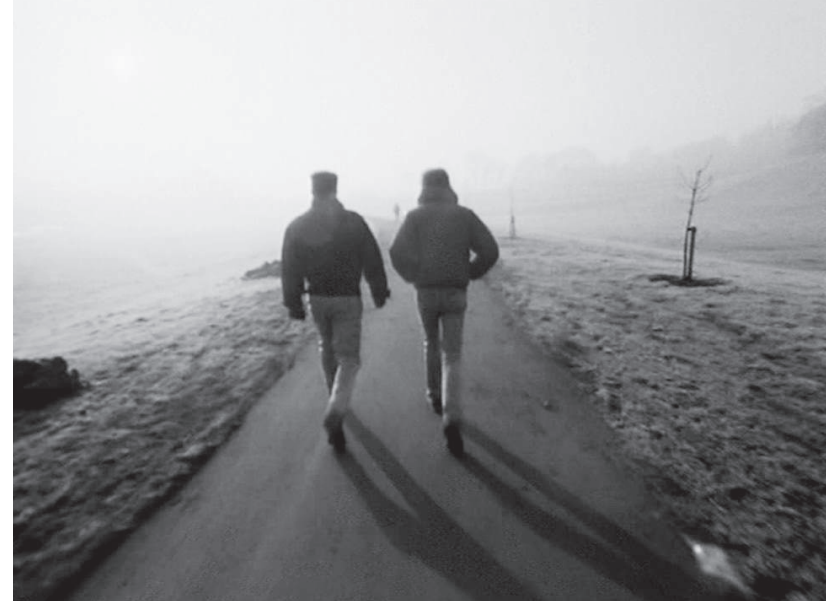

34

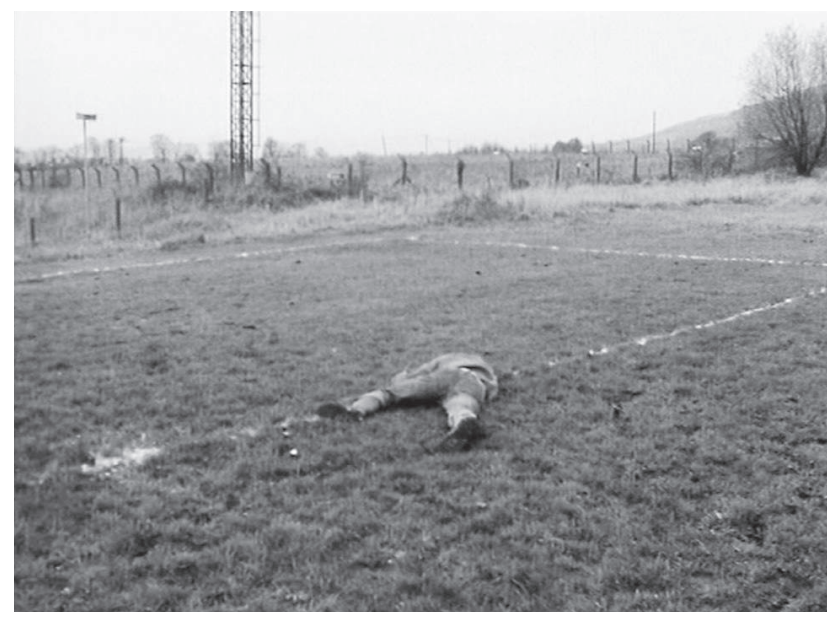

33

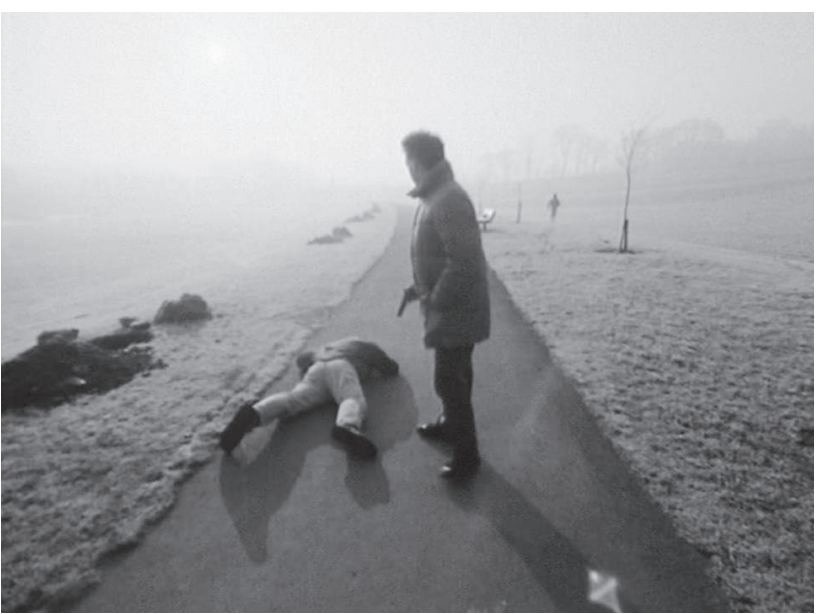

35

nous ne savons rien jusqu'à un endroit désert où il abat quelqu'un de sang froid avec une arme à feu. La structure tripartite telle qu'elle est envisagée dans la conception aristotélicienne du récit est réduite ici à sa plus simple expression: situation banale et calme au début (néanmoins tendue vers une complication en raison de l'allure décidée des protagonistes, puis, une fois que le principe est compris de spectateur, en raison de l'imminence du surgissement de la violence), renversement avec les coups de feu puis retour désenchanté à une forme de tranquillité avec les plans immobiles et rapprochés sur le corps de la victime qui, sous une forme parente des photographies de police documentant une scène de crime, offrent presqu'invariablement une clôture à la séquence. 
Elephant de Van Sant s'inspire notamment du film homonyme au niveau de certains principes de filmage, puisque Clarke suit également, avec une caméra fréquemment située dans le dos des personnages (fig. 31) et perpétuellement en mouvement, les différents tueurs qui parcourent de vastes espaces parsemés de bâtiments suburbains délabrés à la recherche de leur (ou d'une ?) victime. Toute comme Van Sant, le film nerveux et brutal de Clarke explore des espaces, des "zones" qu'il traverse en suivant, à une distance variable, les tueurs qui se précipitent pour accomplir leur sinistre tâche; ainsi trouve-t-on également une scène située sur un terrain de football, dont les lignes blanches qui passent sous les pieds de l'assassin puis sous le corps de la victime semblent quadriller le lieu de la tuerie (fig. 32-33). Lorsque les gens se croisent dans l'Elephant de Clarke - dans des instants où, comme chez Van Sant, une grande importance est accordée à ce qui se déroule dans la profondeur de l'image (fig. 33-35) -, la mort est inévitablement au rendez-vous. Plus encore que chez Van Sant, les personnages demeurent muets; et, ici, nulle musique de Beethoven ni grondements du ciel pour adoucir le drame ou le charger d'un souffle romantique : le film de Clarke s'en tient inflexiblement aux seuls bruits de fond de la circulation sur lesquels se détachent les claquements secs des détonations. L'éviction de toute émotion au profit d'une mécanicité des actes qui confine à l'absurde nous permet de mesurer l'écart qui sépare ce court métrage de facture expérimentale du film de Gus Van Sant: comme le dit Serge Kaganski dans le livret de l'édition DVD MK2 d'Elephant qui comprend également, en supplément, le film de Clarke, "celui de Van Sant est plus sensuel, peuplé de véritables personnages, empli de chair et de fiction». Ce qui donne consistance aux êtres qui traversent les salles du lycée avec sadisme ou effroi tient avant tout à la manière dont le film de Van Sant parvient à nous immerger dans son monde, ne serait-ce, parfois, que l'espace d'un (passionnant) regard. 